\title{
Dermatological Ultrasound in EFSUMB: An application that has come to stay, grow and last
}

The ultrasonographic study of skin and appendages (hair and skin) is a growing recent development in clinical dermatology and aesthetic medicine. New equipment with lineal high frequency probes and highly sensitive Doppler have been key techinical developments that have permitted the use of ultrasound for the study of the heatlhy and pathological skin.

Since pioneer seminal work of Dr Wortsman in Chile, dermatological ultrasound has

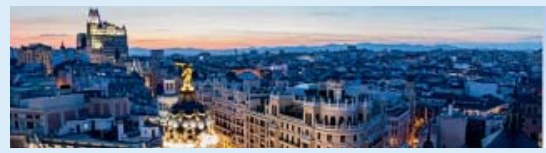

Dermatologic Ultrasound Course - Level 1 EUROSON SCHOOL

Madrid, Spain $\quad 1$ - 2 June 2018

evolved and been introduced in clinical practice by the dermatologic community, mainly in European countries.

Recently the DERMUS group, an independent group that includes dermatologists and radiologists globally have published practice guidelines for this aplication in the AIUM Journal and have set a standard for the practice and teaching of dermatologic ultrasound for the radiologists and clinicians interested in this application. 
From EFSUMB we have created a working group for the development of this application in Europe with the idea of bringing together expertise, investigation and learning to the interested physicians.

With that intention and aiming to create an European consensus on the practice of dermatological ultrasound in Europe under the EFSUMB umbrella, the First Dermatological Ultrasound Euroson School (Level 1) is being organised in which formal theoretical and practical state of the art of this application will be taught by leading experts in this speciality.

Along with the course a formal acreditation on dermatological ultrasound level one by EFSUMB will be offered to participants who succeed in the theoretical and practical evaluation of this course.
This event will take place in the Dermatological Ultrasound Teaching Centre Puerta de Hierro Majadahonda, Madrid, Spain next 1st and 2nd June 2018. For those interested in this new and rapidliy evolving technique this is a great oportunity to learn in depth the fundamentals of the application in the clinical environment.

We hope to see you in Madrid next June. Dr. Fernando Alfageme 